\title{
Completing Partial Proper Colorings using Hall's Condition
}

\author{
Sarah Holliday Jennifer Vandenbussche Erik E. Westlund \\ Department of Mathematics \\ Kennesaw State University \\ Kennesaw, Georgia U.S.A. \\ $\{$ shollid4, jvandenb, ewestlun\}@kennesaw.edu
}

Submitted: May 21, 2014; Accepted: Jun 18, 2015; Published: Jul 1, 2015

Mathematics Subject Classifications: 05C15

\begin{abstract}
In the context of list-coloring the vertices of a graph, Hall's condition is a generalization of Hall's Marriage Theorem and is necessary (but not sufficient) for a graph to admit a proper list-coloring. The graph $G$ with list assignment $L$ satisfies Hall's condition if for each subgraph $H$ of $G$, the inequality $|V(H)| \leqslant \sum_{\sigma \in \mathcal{C}} \alpha(H(\sigma, L))$ is satisfied, where $\mathcal{C}$ is the set of colors and $\alpha(H(\sigma, L))$ is the independence number of the subgraph of $H$ induced on the set of vertices having color $\sigma$ in their lists. A list assignment $L$ to a graph $G$ is called Hall if $(G, L)$ satisfies Hall's condition. A graph $G$ is Hall $m$-completable for some $m \geqslant \chi(G)$ if every partial proper $m$-coloring of $G$ whose corresponding list assignment is Hall can be extended to a proper coloring of $G$. In 2011, Bobga et al. posed the following questions: (1) Are there examples of graphs that are Hall $m$-completable, but not Hall $(m+1)$-completable for some $m \geqslant 3$ ? (2) If $G$ is neither complete nor an odd cycle, is $G$ Hall $\Delta(G)$-completable? This paper establishes that for every $m \geqslant 3$, there exists a graph that is Hall $m$ completable but not Hall $(m+1)$-completable and also that every bipartite planar graph $G$ is Hall $\Delta(G)$-completable.
\end{abstract}

Keywords: vertex coloring; list coloring; partial proper coloring; Hall's condition; Hall $m$-completable.

\section{Introduction}

We investigate a series of questions posed by Bobga et al. [2] regarding the completion of partial proper vertex colorings of finite, simple graphs by using a generalization of Hall's Marriage Theorem applied to list colorings. Throughout, $G$ is a finite, simple graph with vertex set $V(G)$ and edge set $E(G)$. For $U \subseteq V(G)$, we shall use $G[U]$ to denote the subgraph of $G$ induced on $U$. Additionally $\alpha(G), \delta(G), \Delta(G), \chi(G)$, respectively, 
shall denote the independence number, minimum degree, maximum degree, and chromatic number of $G$ respectively. We refer the reader to West [8] for any notations not defined here.

Vizing [7] introduced the notion of list-coloring. It was further developed by Erdös, Rubin, and Taylor [3], and has been studied extensively since. If $\mathcal{C}$ is an infinite set and $\mathcal{L}$ is a set of finite subsets of $\mathcal{C}$ (the color set or color palette), then a list assignment to $G$ is a function $L: V(G) \rightarrow \mathcal{L}$. $L$ is a $k$-assignment to $G$ if $|L(v)| \geqslant k$ for all $v \in V(G)$. Given a list assignment $L$ of $G$, with color set $\mathcal{C}$, an $L$-coloring of $G$ is a function $\phi: V(G) \rightarrow \mathcal{C}$ such that $\phi(v) \in L(v)$ for every vertex $v$. An $L$-coloring $\phi$ is proper if each color class induces an independent set. If $G$ has a proper $L$-coloring, we say $G$ is $L$-colorable.

If $G$ has a proper $L$-coloring for all $k$-assignments $L$, then $G$ is $k$-choosable. The list-chromatic number or choice number of $G$, denoted $\chi_{\ell}(G)$, is

$$
\chi_{\ell}(G)=\min \{k: G \text { is } k \text {-choosable }\} .
$$

Naturally we have the following inequalities:

$$
\chi(G) \leqslant \chi_{\ell}(G) \leqslant \Delta(G)+1 .
$$

In 1990, Hilton and Johnson [5] introduced the following concept (also see [2]), which was a generalization of Philip Hall's 1935 Marriage Theorem applied to list-assignments of graphs. Suppose that $\phi$ is an $L$-coloring of $G$ for some list assignment $L$ with a color palette $\mathcal{C}$ and let $H$ be any subgraph of $G$. For each $\sigma \in \mathcal{C}$, consider $\left.\phi^{-1}(\sigma)\right|_{H}$, the set of all vertices in $H$ given color $\sigma$ under $\phi$, and let $H(\sigma, L)$ be the subgraph of $H$ induced on all vertices of $H$ having $\sigma$ in their lists. Then $\left.\phi^{-1}(\sigma)\right|_{H}$ is an independent set of vertices contained inside $H(\sigma, L)$. Naturally, if $G$ is $L$-colorable, then for every subgraph $H$, we must have

$$
|V(H)|=\sum_{\sigma \in C}\left[\left.\phi^{-1}(\sigma)\right|_{H}\right] \leqslant \sum_{\sigma \in C} \alpha(H(\sigma, L))
$$

This motivated the following definition:

Definition 1 ([5]). The graph $G$ with list assignment $L$, denoted $(G, L)$, satisfies Hall's condition if for each subgraph $H$ of $G$, the inequality

$$
|V(H)| \leqslant \sum_{\sigma \in \mathcal{C}} \alpha(H(\sigma, L)) .
$$

is satisfied.

Observation 2 ([5]). If $G$ has a proper $L$-coloring, then $(G, L)$ satisfies Hall's condition. Also, $(G, L)$ satisfies Hall's condition if and only if $(\mathrm{HC})$ holds for each connected, induced subgraph $H$ of $G$.

Theorem 3 ([5]). If $L$ is a $\chi(G)$-assignment to $G$, then $(G, L)$ satisfies Hall's condition.

Though Hall's Condition is time consuming to verify directly by brute force, we make use of the following observation, which follows from Theorem 2: 
Observation 4. Let $L$ be a list assignment to a graph $G$. If $G-v$ is $L$-colorable for all $v \in V(G)$, then $(G, L)$ satisfies Hall's condition if and only if

$$
|V(G)| \leqslant \sum_{\sigma \in \mathcal{C}} \alpha(G(\sigma, L)) .
$$

It is routine to verify that Hall's condition applied to list-assignments of $K_{n}$ is exactly Hall's Marriage Theorem. In general, however, Hall's condition is not sufficient for $G$ to have an $L$-coloring (see Figure 1). This motivates the study of situations in which this obvious necessary condition is sufficient.

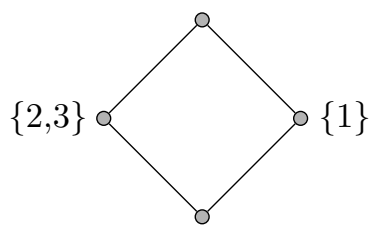

$\{1,3\}$

Figure 1: $(G, L)$ satisfies Hall's condition (see Observation 4 ), yet $G$ is not $L$-colorable.

Definition 5. [5] The Hall number of $G$ is the smallest positive integer $h(G)=k$ such that whenever $L$ is a $k$-assignment to $G$ and $(G, L)$ satisfies Hall's condition, $G$ is $L$-colorable.

In other words, $h(G)$ is the smallest positive integer such that Hall's condition on $k$-assignments is both necessary and sufficient for the existence of a proper $L$-coloring of $G$. Clearly $h(G) \leqslant \chi_{\ell}(G)$.

The following result characterizes graphs with Hall number 1.

Theorem 6 ([5], Hilton et al. [6]). The following statements are equivalent:

1. $h(G)=1$.

2. Every block (maximally 2-connected subgraph) of $G$ is a clique.

3. $G$ contains no induced cycle $C_{n}, n \geqslant 4$, nor an induced copy of $K_{4}-e$.

Graphs with Hall number 2 have been characterized by Eslahchi-Johnson [4], but the characterization is far more complicated.

The completion of a partial coloring of $G$ can be viewed as a list-coloring problem, where the lists on precolored vertices have size one, and the list on any other vertex contains the colors that do not appear on precolored vertices in its neighborhood. In this paper, we study Hall's condition in the context of completions of partial colorings. Let $[m]$ denote the set $\{1, \ldots, m\}$. 
Definition 7. A list assignment $L$ of a graph $G$ is a Hall assignment to $G$ if $(G, L)$ satisfies Hall's condition. For $V_{0} \subseteq V(G)$, a partial proper coloring $\phi: V_{0} \rightarrow[m]$ of a graph $G$ is a Hall m-precoloring if $L_{\phi}$ is a Hall assignment, where

$$
L_{\phi}(x)= \begin{cases}\{\phi(x)\} & \text { if } x \in V_{0} \\ {[m] \backslash\left\{\phi(y): y \in N_{G}(x) \cap V_{0}\right\}} & \text { if } x \notin V_{0} .\end{cases}
$$

A Hall $m$-precoloring $\phi$ is completable if there exists an extension of $\phi$ to a complete proper $m$-coloring of $G$. A graph $G$ is Hall $m$-completable if every Hall $m$-precoloring is completable. A Hall $m$-precoloring that is not completable is incompletable. $G$ is Hall chromatic completable if $G$ is Hall $\chi(G)$-completable and $G$ is total Hall completable if $G$ is Hall $m$-completable for all $m \geqslant \chi(G)$.

The definition of "Hall $m$-completable," was first stated in [2], though different terminology was used. That paper also established the following basic results regarding partial proper $m$-colorings.

Theorem 8 ([2]). Let $\phi: V_{0} \rightarrow[m]$ be a partial proper $m$-coloring of $G$, and let $G^{\prime}=$ $G\left[V \backslash V_{0}\right]$.

1. $G$ is $L_{\phi^{-}}$-colorable if and only if $G^{\prime}$ is $L_{\phi^{-}}$-colorable.

2. $\left(G, L_{\phi}\right)$ satisfies Hall's condition if and only if $\left(G^{\prime}, L_{\phi}\right)$ satisfies Hall's condition.

Observation 9 ([2]). If $m \geqslant \Delta(G)+1$, then every partial proper $m$-coloring of $G$ has a completion, and so every graph $G$ is Hall $m$-completable for all $m \geqslant \Delta(G)+1$.

Observation $10([2]) . G$ is Hall m-completable if and only if every component of $G$ is Hall m-completable.

The following strange result elucidates the subtlety of the Hall number of a graph.

Theorem 11 ([2]). Every bipartite graph is Hall chromatic completable, but for every $m \geqslant 3$, there exists a bipartite graph which is not Hall $m$-completable.

The example given by Bobga et al. had girth four, but in fact their example can be extended to one of arbitrary even girth. Figure 2 shows a $2 n$-cycle $C_{2 n}$ with a list assignment $L$ such that $\left(C_{2 n}, L\right)$ satisfies Hall's condition, but the cycle has no $L$-coloring. Attaching vertices of degree 1 to the vertices of the cycle and coloring the pendant vertices appropriately results in a bipartite graph $G$ with girth $2 n$ and a precoloring $\phi$ such that $\left(G, L_{\phi}\right)$ satisfies Hall's condition, but $\phi$ cannot be extended to a proper coloring of $G$.

Motivated by this strange behavior of bipartite graphs with respect to Hall number, Bobga et al. [2] posed the following question:

Question 1: Are there examples of graphs that are Hall $m$-completable but not Hall $(m+1)$-completable for some $m \geqslant 3$ ?

They also asked the following question, which is a natural analog to Brooks' Theorem:

Question 2: Let $G$ be a connected graph that is neither complete nor an odd cycle. Is it true that $G$ is Hall $\Delta(G)$-completable? 


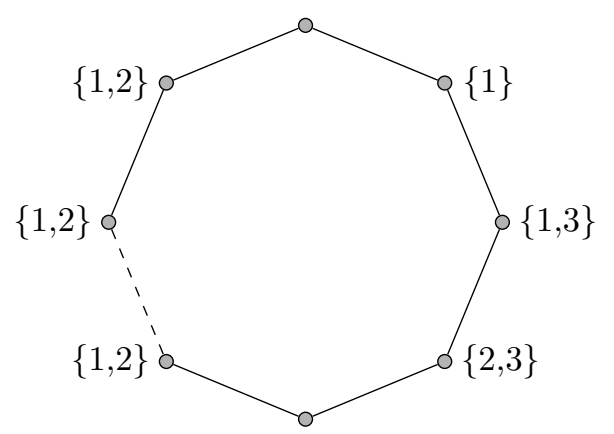

$\{1,2\}$

Figure 2: A Hall 1-assignment $L$ to $C_{2 n}(2 n-3$ vertices all have lists $\{1,2\})$, yet $C_{2 n}$ is not $L$-colorable.

In Section 2, we answer Question 1 in the affirmative. Answering Question 2 seems difficult, but we take a step in that direction by proving in Section 3 that bipartite planar graphs are Hall $\Delta(G)$-completable. Finally, in Section 4 we provide some examples of graphs that are total Hall completable.

\section{A graph that is Hall $m$-completable but not Hall $(m+1)$ - completable}

In this section, we generalize the example in Figure 2 to provide, for all $m \geqslant 2$, graphs that are Hall $m$-completable but not Hall $(m+1)$-completable. We shall use $G_{m}$ to denote the graph of order $m+2$ formed by subdividing an edge of $K_{m+1}$.

Lemma 12. There exists a list assignment $L$ of $V\left(G_{m}\right)$ with colors chosen from $[m+1]$ such that $\left(G_{m}, L\right)$ satisfies Hall's condition, but $G_{m}$ is not L-colorable.

Proof. Let $x y$ be the subdivided edge, and let $z$ be the new vertex of degree two. Let $v_{1}, \ldots, v_{m-1}$ be the remaining vertices of $G_{m}$. Consider the following list assignment:

$$
L(v)= \begin{cases}\{1,2\} & \text { if } v=x \\ \{1,3\} & \text { if } v=y \\ \{1\} & \text { if } v=z \\ \{2,3, \ldots, m+1\} & \text { otherwise }\end{cases}
$$

Note that $G_{m}$ is not $L$-colorable, since in any proper $L$-coloring $\gamma, \gamma(x)=2$ and $\gamma(y)=3$, leaving only $m-2$ colors available for the clique $\left\{v_{1}, v_{2}, \ldots, v_{m-1}\right\}$. It remains to verify that $\left(G_{m}, L\right)$ satisfies $(\mathrm{HC})$. Observe that

$$
\left|V\left(G_{m}\right)\right| \leqslant \sum_{\sigma \in[m+1]} \alpha\left(G_{m}(\sigma, L)\right) .
$$


Since $G_{m}-v$ is $L$-colorable for any $v \in V\left(G_{m}\right)$, by Observation $4,\left(G_{m}, L\right)$ satisfies Hall's condition.

Note that when $m=2$, the graph $G_{m}$ is $C_{4}$, so $G_{m}$ is a generalization of the example given by Bobga et al. in [2].

Let $G_{m}^{\prime}$ be the graph obtained from $G_{m}$ by adding $m$ pendant vertices to $z, m-1$ pendant vertices to $x$ and $y$, and one pendant vertex to each $v_{i}, 1 \leqslant i \leqslant m-1$. Now precoloring the pendant vertices of $G_{m}^{\prime}$ appropriately yields the list assignment $L$ above on the vertices of $G_{m}$. This assignment on $G_{m}$ together with the singleton lists on the precolored pendant vertices satisfies Hall's condition by Lemma 12 and Theorem 8, yet the precoloring will not extend to a proper $(m+1)$-coloring of $G_{m}^{\prime}$ (see Figure 3 ).

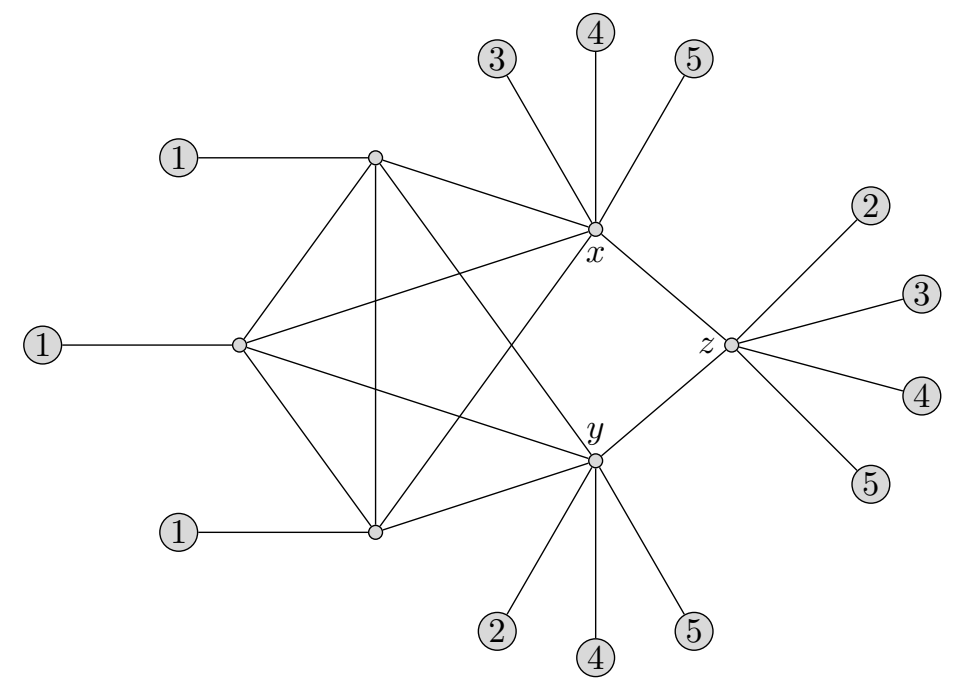

Figure 3: The Hall 4-completable graph $G_{4}^{\prime}$ from Theorem 13 with an incompletable Hall 5-precoloring.

Theorem 13. For all $m \geqslant 2$, there exists a graph that is Hall $m$-completable but not Hall $(m+1)$-completable.

Proof. We show that $G_{m}^{\prime}$ is such a graph. Lemma 12 verifies that $G_{m}^{\prime}$ is not Hall $(m+1)$ completable, hence it remains to show that $G_{m}^{\prime}$ is Hall $m$-completable.

Let $\phi: V_{0} \rightarrow[m]$ be a Hall $m$-precoloring of $G_{m}^{\prime}$. If $\phi$ extends to a proper $m$ coloring of $G_{m} \subseteq G_{m}^{\prime}$, then $\phi$ also extends to a proper $m$-coloring of $G_{m}^{\prime}$, since every $v \in V\left(G_{m}^{\prime}\right) \backslash V\left(G_{m}\right)$ has only one neighbor in $G_{m}$ and $m \geqslant 2$. Therefore we verify that $\phi$ extends to a proper $m$-coloring of $G_{m}$.

By assumption,

$$
m+2=\left|V\left(G_{m}\right)\right| \leqslant \sum_{\sigma \in[m]} \alpha\left(G_{m}\left(\sigma, L_{\phi}\right)\right) .
$$

Since $\alpha\left(G_{m}\right)=2$, no color can contribute more than two to the right side of this inequality. All independent sets of size two in $G_{m}$ are of the form $\{x, y\}$ or $\left\{z, v_{i}\right\}$ for 
some $1 \leqslant i \leqslant m-1$. Since $\left|V\left(G_{m}\right)\right|=m+2$, there must be at least two such independent sets, each of whose vertices have non-disjoint lists. If $L(x) \cap L(y)=\varnothing$, then $L_{\phi}$ restricted to $G_{m}-z$ fails Hall's condition because no color can contribute more than one to the righthand side of (HC). Similarly, if $L(z) \cap L\left(v_{i}\right)=\varnothing$ for all $1 \leqslant i \leqslant m-1$, then $L_{\phi}$ restricted to $G_{m}-x$ fails Hall's condition. Furthermore, if $\left|(L(x) \cap L(y)) \cup\left(L(z) \cap L\left(v_{i}\right)\right)\right|=1$ for every $1 \leqslant i \leqslant m-1$, then only one color can contribute two to the right side of the inequality, a contradiction. Therefore we may assume without loss of generality that $1 \in L_{\phi}(x) \cap L_{\phi}(y)$ and $2 \in L_{\phi}(z) \cap L_{\phi}\left(v_{1}\right)$.

Consider the clique $H=\left\{x, v_{1}, v_{2}, \ldots, v_{m-1}\right\}$. Since $\left(H, L_{\phi}\right)$ satisfies (HC) and cliques have Hall number 1 by Theorem $6, H$ has an $L_{\phi}$-coloring. If there exists such a coloring $\gamma$ with $\gamma(x)=1$, then $\gamma$ can be extended to $G_{m}$ via $\gamma(y)=1$ and $\gamma(z)=2$. Hence we may assume that every $L_{\phi}$-coloring of $H$ uses color 1 on some $v_{i}$.

Consider $L_{\phi}^{\prime}$ defined by

$$
L_{\phi}^{\prime}(v)= \begin{cases}\{1\} & \text { if } v=x \\ L_{\phi}(v) & \text { otherwise }\end{cases}
$$

By assumption, $H$ is not $L_{\phi}^{\prime}$-colorable. (Note that this implies that $x$ is not precolored by $\phi$.) Again since the Hall number of $H$ is 1 , there exists a subgraph $H^{\prime}$ of $H$ having largest order such that $\left(H^{\prime}, L_{\phi}^{\prime}\right)$ fails to satisfy $(\mathrm{HC})$.

Recall that $V_{0}$ is the set of vertices precolored by $\phi$.

Claim 1: There exists $\sigma \in L(x)$ such that if $v_{i} \in V\left(H^{\prime}\right)$ and $v_{i} \notin V_{0}$, then the pendant neighbor of $v_{i}$ in $G_{m}^{\prime}$ is precolored with $\sigma$. Consequently, for any $v_{i}, v_{j} \in V\left(H^{\prime}\right) \backslash V_{0}$, $L_{\phi}^{\prime}\left(v_{i}\right)=L_{\phi}^{\prime}\left(v_{j}\right)$.

Proof of Claim 1: Since $\alpha\left(H^{\prime}\right)=1$ and $\left(H^{\prime}, L_{\phi}^{\prime}\right)$ fails to satisfy (HC) on $H^{\prime}$,

$$
\left|\bigcup_{v \in V\left(H^{\prime}\right)} L_{\phi}^{\prime}(v)\right| \leqslant\left|V\left(H^{\prime}\right)\right|-1 .
$$

But $\left(K, L_{\phi}\right)$ satisfies (HC) on every subgraph $K$ of $G_{m}^{\prime}$, so

$$
\left|\bigcup_{v \in V\left(H^{\prime}\right)} L_{\phi}(v)\right| \geqslant\left|V\left(H^{\prime}\right)\right| .
$$

Since $L_{\phi}^{\prime}$ and $L_{\phi}$ differ only at the vertex $x$, there is some color $\sigma \in L(x)$ that does not appear on $L_{\phi}\left(v_{i}\right)$ for any $v_{i} \in V\left(H^{\prime}\right)$. By the definition of $L_{\phi}$, for any $v \in V\left(G_{m}\right) \backslash V_{0}$, if $\sigma \notin L_{\phi}(v)$, then $\phi(w)=\sigma$ for some $w \in N(v)$. Each $v_{i} \in V\left(G_{m}^{\prime}\right)$ has exactly one neighbor $v_{i}^{\prime}$ for which $v_{i}^{\prime} \notin N(x)$ (namely the attached pendant vertex). Therefore for each $v_{i} \in V\left(H^{\prime}\right)$, either $v_{i} \in V_{0}$, or its pendant neighbor $v_{i}^{\prime}$ is such that $\phi\left(v_{i}^{\prime}\right)=\sigma$. Hence for any $v_{i} \in V\left(H^{\prime}\right) \backslash V_{0}, L_{\phi}^{\prime}\left(v_{i}\right)=L_{\phi}\left(v_{i}\right)=[m] \backslash X \backslash\{\sigma\}$, where $X$ is the set of all colors appearing on precolored vertices of the $(m-1)$-clique $\left\{v_{1}, v_{2}, \ldots, v_{m-1}\right\}$.

Claim 2: Some $v_{i} \in V\left(H^{\prime}\right)$ is not in $V_{0}$. 
Proof of claim 2: Suppose otherwise. Since $H^{\prime}$ fails (HC) and $\alpha\left(H^{\prime}\right)=1$, there must be at most $\left|V\left(H^{\prime}\right)\right|-1$ colors on the lists of $V\left(H^{\prime}\right)$. All precolored vertices of $H^{\prime}$ will contribute a distinct color, and since $1 \in L_{\phi}(x)$, none of them can be colored with 1 . Hence the color 1 also contributes to the right side of inequality (HC), a contradiction.

Claim 3: $V\left(H^{\prime}\right)=\left\{v_{1}, \ldots, v_{m-1}\right\}$.

Proof of claim 3: Suppose otherwise, that $v_{j} \notin V\left(H^{\prime}\right)$ for some $j \in[m-1]$. By the maximality of $H^{\prime}$, if $H^{\prime \prime}$ is the subgraph of $G_{m}^{\prime}$ induced on $V\left(H^{\prime}\right) \cup\left\{v_{j}\right\}$, then $\left(H^{\prime \prime}, L_{\phi}^{\prime}\right)$ satisfies (HC), so

$$
\left|V\left(H^{\prime}\right)\right|+1=\left|V\left(H^{\prime \prime}\right)\right| \leqslant \sum_{\sigma \in C} \alpha\left(H^{\prime \prime}\left(\sigma, L_{\phi}^{\prime}\right)\right) .
$$

Furthermore, $\alpha\left(H^{\prime \prime}\right)=1$, and so

$$
\left|V\left(H^{\prime}\right)\right|+1=\left|V\left(H^{\prime \prime}\right)\right| \leqslant\left|\bigcup_{v \in V\left(H^{\prime \prime}\right)} L_{\phi}^{\prime}(v)\right| .
$$

Since $H^{\prime}$ fails (HC),

$$
\left|V\left(H^{\prime}\right)\right|>\sum_{\sigma \in C} \alpha\left(H^{\prime}\left(\sigma, L_{\phi}^{\prime}\right)\right),
$$

and hence $V\left(H^{\prime \prime}\right) \backslash V\left(H^{\prime}\right)=\left\{v_{j}\right\}$ implies that

$$
\left|L_{\phi}^{\prime}\left(v_{j}\right) \backslash \bigcup_{v \in V\left(H^{\prime}\right)} L_{\phi}^{\prime}(v)\right| \geqslant 2 .
$$

But if $v_{j} \in V_{0},\left|L_{\phi}^{\prime}\left(v_{j}\right)\right|=1$; if $v_{j} \notin V_{0}$, then by Claim $1, \sigma$ can be the only element of $L_{\phi}^{\prime}\left(v_{j}\right)$ not in $\bigcup_{v \in V\left(H^{\prime}\right)} L_{\phi}^{\prime}(v)$. In either case,

$$
\left|L_{\phi}^{\prime}\left(v_{j}\right) \backslash \bigcup_{v \in V\left(H^{\prime}\right)} L_{\phi}^{\prime}(v)\right| \leqslant 1
$$

a contradiction. Hence the claim is established.

Now Claim 1 and Claim 3 imply that there is a color $\sigma \in L_{\phi}(x)$ such that for all $i \in[m-1]$, either $v_{i} \in V_{0}$ or the pendant neighbor $v_{i}^{\prime}$ of $v_{i}$ is such that $\phi\left(v_{i}^{\prime}\right)=\sigma$. A symmetric argument applied to $y$ yields some color $\tau \in L_{\phi}(y)$ such that for all $i \in[m-1]$, either $v_{i} \in V_{0}$ or the pendant neighbor $v_{i}^{\prime}$ of $v_{i}$ is such that $\phi\left(v_{i}^{\prime}\right)=\tau$. By Claim 2, there is some $v_{i} \in V\left(H^{\prime}\right) \backslash V_{0}$, and hence there is a vertex $v_{i}^{\prime}$ that is precolored; hence $\sigma=\tau$, and $\sigma \in L_{\phi}(y)$. Since $\sigma$ did not appear in any list in $V\left(H^{\prime}\right)$ and we assumed $2 \in L\left(v_{1}\right)$, $\sigma \neq 2$. Hence $\phi$ can be extended by coloring $x$ and $y$ with color $\sigma$ and $z$ with color 2 , and giving $v_{1}, \ldots, v_{m-1}$ an $(m-1)$-coloring guaranteed by Hall's condition. 


\section{Hall $\Delta(G)$-completable bipartite graphs}

In this section, we prove the following:

Theorem 14. If $G$ is a bipartite graph with $h(G) \leqslant 3$, then $G$ is Hall $\Delta(G)$-completable.

Before proving the theorem, we give several corollaries. Since $h(G) \leqslant \chi_{\ell}(G)$, we have the following:

Corollary 15. If $G$ is a 3-choosable bipartite graph, then $G$ is Hall $\Delta(G)$-completable.

Alon and Tarsi [1] proved that every bipartite planar graph is 3-choosable.

Corollary 16. If $G$ is a bipartite planar graph, then $G$ is Hall $\Delta(G)$-completable.

Recall that if $G$ and $H$ are graphs, then the cartesian product of $G$ and $H$ is the graph $G \square H$ having vertex set $V(G) \times V(H)$ and edge set $E(G \square H)$ defined as

$$
\left\{\left\{\left(g_{1}, h_{1}\right),\left(g_{2}, h_{2}\right)\right\}: h_{1}=h_{2} \text { and }\left\{g_{1}, g_{2}\right\} \in E(G) \text { or } g_{1}=g_{2} \text { and }\left\{h_{1}, h_{2}\right\} \in E(H)\right\} \text {. }
$$

Corollary 17. If $\min \{n, k\} \geqslant 4$, then the cartesian product $P_{n} \square P_{k}$ is Hall m-completable if and only if $m \neq 3$.

Proof. Let $\min \{n, k\} \geqslant 4 . \quad G=P_{n} \square P_{k}$ is a bipartite graph, and thus it is Hall 2completable. Since it is planar with maximum degree 4, Corollary 16 implies it is Hall 4-completable, and Theorem 9 implies it is Hall $m$-completable for all $m \geqslant 5$. Finally we claim there exists an incompletable Hall 3-precoloring of $G$. Consider the precoloring $\phi: V_{0} \rightarrow[3]$ shown in Figure 4, where $\left|V_{0}\right|=7$. Note $L_{\phi}\left(v_{1}\right)=\{1,2\}, L_{\phi}\left(v_{2}\right)=\{1,3\}$, $L_{\phi}\left(v_{3}\right)=\{3\}$, and $L_{\phi}\left(v_{4}\right)=\{2,3\}$, and for all $v \in V \backslash\left(V_{0} \cup\left\{v_{1}, v_{2}, v_{3}, v_{4}\right\}\right)$ we have that $\left|L_{\phi}(v)\right| \geqslant 2$. If $H$ is any connected, induced subgraph of $G^{\prime}=G\left[V \backslash V_{0}\right]$, then

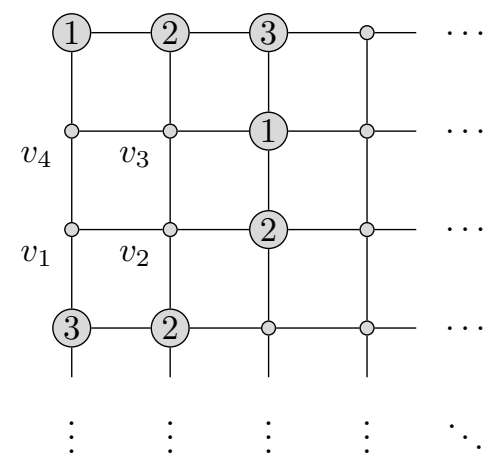

Figure 4: The incompletable Hall 3-precoloring $\phi$ from Corollary 17.

either $V(H) \cap\left\{v_{1}, v_{2}, v_{3}, v_{4}\right\}=\varnothing$ or $H$ is a subgraph of the 4-cycle induced by vertices $\left\{v_{1}, v_{2}, v_{3}, v_{4}\right\}$. In the former case, $L_{\phi}$ restricted to $H$ is a 2 -assignment, and thus a Hall assignment by Theorem 3 since $H$ is bipartite, so $\left(H, L_{\phi}\right)$ satisfies $(\mathrm{HC})$. In the latter case $\left(H, L_{\phi}\right)$ satisfies $(\mathrm{HC})$ by Observation 4 . Hence, $\left(G, L_{\phi}\right)$ satisfies $(\mathrm{HC})$ but clearly $\phi$ is not extendable. 
By the same argument, we have the following.

Corollary 18. If $n \geqslant 2$ or $k \geqslant 4$, then the generalized prism graphs $C_{2 n} \square P_{k}$ are Hall $m$-completable if and only if $m \neq 3$.

The following will be used in the proof of Theorem 14 .

Definition 19. Let $\phi: V_{0} \rightarrow[m]$ be a precoloring of $G$ with list assignment $L_{\phi}$ and let $\theta: V_{1} \rightarrow[m]$ be a precoloring of $G^{\prime}=G\left[V \backslash V_{0}\right]$ with list assignment $L_{\theta}$. The coloring $\theta$ (or the list assignment $L_{\theta}$ ) respects $L_{\phi}$ if

$$
L_{\theta}(x)= \begin{cases}\theta(x) \in L_{\phi}(x) & \text { if } x \in V_{1} \\ L_{\phi}(x) \backslash\left\{\theta(y): y \in N_{G^{\prime}}(x) \cap V_{1}\right\} & \text { if } x \in G^{\prime}(V) \backslash V_{1}\end{cases}
$$

where $N_{G^{\prime}}(x)$ denotes the open neighborhood of $x$ in $G^{\prime}$.

If $\theta$ respects $\phi$, then $\phi \cup \theta$ is a proper precoloring of $V_{0} \cup V_{1} \subseteq V(G)$. The following observation follows directly from definitions and the fact that $L_{\phi \cup \theta}(x)=L_{\theta}$ for any $x \notin V_{0} \cup V_{1}$ :

Observation 20. If $\phi$ is a Hall m-precoloring of $G$ and if $\theta$ is a Hall m-precoloring of $G^{\prime}$ respecting $L_{\phi}$, then $\phi \cup \theta$ is a Hall m-precoloring of $G$ that extends $\phi$.

We are now ready to prove Theorem 14 .

Proof. Out of all incompletable Hall $\Delta(G)$-precolorings of $G$, let $\phi: V_{0} \rightarrow[\Delta(G)]$ precolor a maximal number of vertices and let $G^{\prime}=G\left[V \backslash V_{0}\right]$. Clearly, $V \neq V_{0}$ and $\left.L_{\phi}\right|_{G^{\prime}}$ is a Hall assignment but by Theorem $8, G^{\prime}$ is not $L_{\phi^{-}}$colorable. Furthermore, $\delta\left(G^{\prime}\right) \geqslant 1$, for any isolated vertex of $G^{\prime}$ could be colored, contradicting the maximality of $\phi$. Suppose that $v \in V\left(G^{\prime}\right)$ and $\left|N_{G}(v) \cap V_{0}\right|=j$. Then

$$
\left|L_{\phi}(v)\right| \geqslant \Delta(G)-j \geqslant \operatorname{deg}_{G}(v)-j=\operatorname{deg}_{G^{\prime}}(v) .
$$

Therefore, for all $v \in V\left(G^{\prime}\right)$

$$
\Delta(G) \geqslant\left|L_{\phi}(v)\right| \geqslant \operatorname{deg}_{G^{\prime}}(v) \geqslant 1 .
$$

By hypothesis, $h\left(G^{\prime}\right) \leqslant h(G) \leqslant 3$ and $L_{\phi}$ is a Hall 1 -assignment to $G$, and thus to $G^{\prime}$. Since $\left.L_{\phi}\right|_{G^{\prime}}$ is a Hall $\delta\left(G^{\prime}\right)$-assignment, if $\delta\left(G^{\prime}\right) \geqslant h\left(G^{\prime}\right)$, then $G^{\prime}$ is $L_{\phi^{\prime}}$-colorable, which contradicts the choice of $\phi$. Therefore, we may assume $1 \leqslant \delta\left(G^{\prime}\right)<h\left(G^{\prime}\right) \leqslant 3$. Consider the set

$$
U=\left\{x \in V\left(G^{\prime}\right): x \text { is not contained in any cycle of } G^{\prime}\right\} .
$$

Claim 1: $|U|=0$.

Proof of Claim 1: Assume otherwise. If $U=V\left(G^{\prime}\right)$, then $G^{\prime}$ is a forest and $h\left(G^{\prime}\right)=1$, a contradiction. Thus $U \neq V\left(G^{\prime}\right)$ and $|U|>0$. Let $F=G^{\prime}[U]$ be the forest with connected components $\left\{F_{1}, \ldots, F_{t}\right\}$ where $t \geqslant 1$. Define

$$
N\left(F_{i}\right)=\left\{v \in V\left(G^{\prime}\right) \backslash U:\{v, y\} \in E\left(G^{\prime}\right) \text { for some } y \in V\left(F_{i}\right)\right\}
$$


and let $\cup_{i=1}^{t} N\left(F_{i}\right)$ be the vertex boundary of $F$. Since the vertices of $F$ are not contained in cycles, any vertex $x$ in the vertex boundary of $F$ is adjacent to at most one vertex in any component of $F$. Furthermore, since $x \notin U, x$ is adjacent to at least two vertices in $V\left(G^{\prime}\right) \backslash U$. Therefore if $x$ is connected to $s$ of the components of $F$, then $\operatorname{deg}_{G^{\prime}}(x) \geqslant$ $s+2 \geqslant 3$.

Note that $h(F)=1$ and $\left.L_{\phi}\right|_{F}$ is a Hall 1-assignment, thus $F$ is $L_{\phi}$-colorable. Let $\theta: V(F) \rightarrow[\Delta(G)]$ be any partial proper $\Delta(G)$-coloring of $G^{\prime}$ that respects $L_{\phi}$ and consider $G^{\prime \prime}=G^{\prime}-F$. For any $x \in V\left(G^{\prime \prime}\right)$, if $x$ is not in the vertex boundary of $F$, then

$$
\left|L_{\theta}(x)\right|=\left|L_{\phi}(x)\right| \geqslant \operatorname{deg}_{G^{\prime}}(x) \geqslant 2
$$

and if $x$ is in the vertex boundary of $F$, then

$$
\left|L_{\theta}(x)\right| \geqslant\left|L_{\phi}(x)\right|-1 \geqslant \operatorname{deg}_{G^{\prime}}(x)-1 \geqslant 3-1=2 .
$$

Therefore $\left.L_{\theta}\right|_{G^{\prime \prime}}$ is a 2-assignment to the bipartite graph $G^{\prime \prime}$ that respects $L_{\phi}$. But any 2-assignment to a bipartite graph is a Hall assignment. Therefore, by Observation 20, $\phi$ can be extended to an incompletable Hall $\Delta(G)$-precoloring of the vertices $V_{0} \cup V(F)$, a contradiction to the maximality of $\phi$, and Claim 1 is established.

Therefore $|U|=0$, and so $\delta\left(G^{\prime}\right)=2<3=h\left(G^{\prime}\right)$. Hence $L_{\phi}$ is a Hall 2-assignment to $G^{\prime}$. Consider the set

$$
T=\left\{x \in V\left(G^{\prime}\right): \operatorname{deg}_{G^{\prime}}(x)=2\right\} .
$$

Note that $T \neq V\left(G^{\prime}\right)$; otherwise each component of $G^{\prime}$ is a cycle, implying $h\left(G^{\prime}\right)=2$, a contradiction. If $T=\varnothing$, then $\delta\left(G^{\prime}\right) \geqslant 3$, which is again a contradiction.

Therefore we may assume that $T \neq V\left(G^{\prime}\right)$ and $T \neq \varnothing$. Let $D=G^{\prime}[T]$ with components $\left\{D_{1}, \ldots, D_{j}\right\}$. For each $i \in\{1, \ldots, j\}, D_{i}$ is either a path or a cycle, so $h(D) \leqslant 2$. The restriction $\left.L_{\phi}\right|_{D}$ is a Hall 2-assignment and hence $D$ is $L_{\phi^{-}}$colorable. If some $D_{i}$ is a cycle, then $D_{i}$ can be $L_{\phi}$-colored without affecting the lists on any other vertices of $G^{\prime}$, contradicting the maximality of $\phi$. Hence each $D_{i}$ is a path. As before, define

$$
N\left(D_{i}\right)=\left\{v \in V\left(G^{\prime}\right) \backslash T:\{v, y\} \in E\left(G^{\prime}\right) \text { for some } y \in V\left(D_{i}\right)\right\}
$$

Let $v$ be a fixed element in $\bigcup_{i=1}^{j} N\left(D_{i}\right)$ and consider the neighborhood $N_{G^{\prime}}(v)$ of $v$ in $G^{\prime}$. We consider two cases.

Case 1: $\left|\boldsymbol{N}_{\boldsymbol{G}^{\prime}}(\boldsymbol{v}) \cap \boldsymbol{V}\left(\boldsymbol{D}_{\boldsymbol{i}}\right)\right|=\mathbf{1}$ for some $i \in\{1, \ldots, j\}$. Here $D_{i}$ is a path

$$
D_{i}=x_{1}, x_{2}, \ldots, x_{k}
$$

where $k \geqslant 1$ and $\left\{v, x_{1}\right\} \in E\left(G^{\prime}\right),\left\{u, x_{k}\right\} \in E\left(G^{\prime}\right)$ for some $u \in N\left(D_{i}\right)$, with $v \neq u$. Let $\theta: V\left(D_{i}\right) \rightarrow[\Delta(G)]$ be partial proper coloring of $G^{\prime}$ that respects $L_{\phi}$ and let $G^{\prime \prime}=$ $G^{\prime}\left[V\left(G^{\prime}\right) \backslash V\left(D_{i}\right)\right]=G^{\prime}-D_{i}$. Clearly, if $x \notin\{u, v\}$ then

$$
\left|L_{\theta}(x)\right|=\left|L_{\phi}(x)\right| \geqslant \operatorname{deg}_{G^{\prime}}(x) \geqslant 2
$$

and if $x \in\{u, v\}$ then

$$
\left|L_{\theta}(x)\right| \geqslant\left|L_{\phi}(x)\right|-1 \geqslant \operatorname{deg}_{G^{\prime}}(x)-1 \geqslant 3-1=2 .
$$


Therefore $L_{\theta}$ is a 2-assignment to $G^{\prime \prime}$, and the assignment is Hall because $G^{\prime \prime}$ is bipartite. By Observation 20, $\phi$ could be extended to an incompletable Hall $\Delta(G)$-precoloring of $V_{0} \cup V\left(D_{i}\right)$, a contradiction to the maximality of $\phi$.

Case 2: $\left|\boldsymbol{N}_{G^{\prime}}(\boldsymbol{v}) \cap \boldsymbol{V}\left(\boldsymbol{D}_{\boldsymbol{i}}\right)\right| \neq \mathbf{1}$ for any $i \in\{1, \ldots, j\}$. Then $N_{G^{\prime}}(v)$ contains $2 d$ vertices of $V(D)$, namely the endpoints of $d$ paths in $\left\{D_{1}, \ldots, D_{j}\right\}$. We consider two subcases.

1. $\boldsymbol{d}=1$. Here $N_{G^{\prime}}(v)$ contains exactly two vertices of degree two in $V\left(D_{i}\right)$ for some $i \in\{1, \ldots, j\}$, say $x_{1}$ and $x_{k}$ where

$$
D_{i}=x_{1}, x_{2}, \ldots, x_{k}
$$

with $k \geqslant 2$, and $\left\{v, x_{1}\right\},\left\{v, x_{k}\right\} \in E\left(G^{\prime}\right)$. Then $\operatorname{deg}_{G^{\prime}}(y) \geqslant 3$ for any $y \in N_{G^{\prime}}(v) \backslash$ $\left\{x_{1}, x_{k}\right\}$. Note $L_{\phi}$ restricted to $G^{\prime}\left[V\left(D_{i}\right) \cup\{v\}\right]$ is a Hall 2-assignment. Let $\theta$ : $V\left(D_{i}\right) \cup\{v\} \rightarrow[\Delta(G)]$ be a partial proper coloring that respects $L_{\phi}$ and let $G^{\prime \prime}=$ $G^{\prime}\left[V\left(G^{\prime}\right) \backslash\left\{V\left(D_{i}\right) \cup\{v\}\right\}\right]$. If $x \in V\left(G^{\prime \prime}\right) \backslash N_{G^{\prime}}(v)$, then

$$
\left|L_{\theta}(x)\right|=\left|L_{\phi}(x)\right| \geqslant \operatorname{deg}_{G^{\prime}}(x) \geqslant 2
$$

and if $x \in V\left(G^{\prime \prime}\right) \cap N_{G^{\prime}}(v)$, then

$$
\left|L_{\theta}(x)\right| \geqslant\left|L_{\phi}(x)\right|-1 \geqslant \operatorname{deg}_{G^{\prime}}(x)-1 \geqslant 3-1=2 .
$$

Therefore, $L_{\theta}$ is a 2-assignment to $G^{\prime \prime}$, which is Hall because $G^{\prime \prime}$ is bipartite. By Observation 20, $\phi$ could be extended to an incompletable Hall $\Delta(G)$-precoloring of $V_{0} \cup V\left(D_{i}\right) \cup\{v\}$, a contradiction to the maximality of $\phi$.

2. $\boldsymbol{d} \geqslant 2$. Let $D_{i}=x_{1}, \ldots, x_{k}$ and $D_{h}=y_{1}, \ldots, y_{s}$ where $\left\{x_{1}, x_{k}, y_{1}, y_{s}\right\} \subseteq N_{G^{\prime}}(v)$. Let $\theta: V\left(D_{i}\right) \rightarrow[\Delta(G)]$ be partial proper coloring that respects $L_{\phi}$ and let $G^{\prime \prime}=$ $G^{\prime}\left[V\left(G^{\prime}\right) \backslash V\left(D_{i}\right)\right]=G^{\prime}-D_{i}$. For any $x \in V\left(G^{\prime \prime}\right)$, if $x \neq v$ then

$$
\left|L_{\theta}(x)\right|=\left|L_{\phi}(x)\right| \geqslant \operatorname{deg}_{G^{\prime}}(x) \geqslant 2 .
$$

Further,

$$
\left|L_{\theta}(v)\right| \geqslant\left|L_{\phi}(v)\right|-2 \geqslant \operatorname{deg}_{G^{\prime}}(v)-2 \geqslant 4-2=2 .
$$

Therefore $L_{\theta}$ is a 2-assignment to $G^{\prime \prime}$, which is Hall because $G^{\prime \prime}$ is bipartite, again a contradiction to the maximality of $\phi$.

This final contradiction implies that there cannot exist any incompletable Hall $\Delta(G)$ precoloring of $G$. Hence, $G$ is Hall $\Delta(G)$-completable. 


\section{Total Hall completable graphs}

In this section, we briefly discuss graphs that are total Hall completable, i.e., Hall $m$ completable for all $m \geqslant \chi$. Total Hall completable graphs are of particular interest in this area of precoloring extensions. One can view total Hall completable graphs as those for which extending precolorings is "easy." As long as the obviously necessary Hall's condition is not violated, any precoloring of a total Hall completable graph $\mathrm{G}$ with at least $\chi(G)$ colors can be extended to a coloring of the entire graph. We present some preliminary results in this area.

Theorem 21 ([2]). If $G$ is an odd cycle, or complete multipartite, or a graph in which every block is a clique, then $G$ is total Hall completable.

Corollary 22. If $G$ is a tree or a cycle, then $G$ is total Hall completable.

Proof. If $G$ is a tree or an odd cycle then we are done by Theorem 21. If $G$ is an even cycle, then $G$ is total Hall completable by Theorems 11 and 9 .

The following results add to the class of total Hall completable graphs. The following is a corollary to Theorem 14 .

Corollary 23. The prism graphs $C_{2 k} \square P_{2}$ and the ladder graphs $P_{n} \square P_{2}$ are total Hall completable for all $k \geqslant 1$ and $n \geqslant 2$.

The following will be used in the proof of Theorem 25 .

Lemma 24. If $G$ has order $n$ and girth $r \geqslant 5$, and $\phi: V_{0} \rightarrow[m]$ is any Hall $m$-precoloring where $\left|V_{0}\right|>n-r$, then $\phi$ can be extended to a proper coloring of $G$.

Proof. By Theorem 8, $G$ will be $L_{\phi^{-}}$-colorable if and only if $G^{\prime}=G\left[V \backslash V_{0}\right]$ is $L_{\phi^{-}}$-colorable. The restriction of $L_{\phi}$ to $G^{\prime}$ satisfies Hall's condition and $G^{\prime}$ has order $n^{\prime}=n-\left|V_{0}\right|<r$. Thus, $G^{\prime}$ possesses no induced $C_{n}, n \geqslant 4$, nor $K_{4}-e$, and so $h\left(G^{\prime}\right)=1$ by Theorem 6 , and so $G^{\prime}$, and thus $G$, is $L_{\phi^{-}}$colorable.

Theorem 25. The Petersen graph is total Hall completable.

Proof. Let $G$ be the Petersen graph. As $\chi(G)=3=\Delta(G)$ we need only show that $G$ is Hall 3-completable. We shall prove by maximal counterexample. Suppose that out of all incompletable Hall 3-precolorings, $\phi: V_{0} \rightarrow[3]$ precolors the largest number of vertices. Let $G^{\prime}=G\left[V \backslash V_{0}\right]$. By assumption, $L_{\phi}$ is a Hall 1-assignment to $G^{\prime}$ that cannot be extended, and note that $\left|L_{\phi}(v)\right| \geqslant \operatorname{deg}_{G^{\prime}}(v)$ for all $v \in V\left(G^{\prime}\right)$. By the maximality of $\left|V_{0}\right|$, $G^{\prime}$ contains no component that has Hall number 1 ; in particular, no component of $G^{\prime}$ is a tree.

Clearly $\left|V_{0}\right|>1$, since any precoloring of a single vertex can be extended. If $\left|V_{0}\right| \geqslant 6$, then we obtain a contradiction by Lemma 24 . Note the only cycles $G^{\prime}$ may possess are $C_{5}, C_{6}, C_{8}$, and $C_{9}$. We consider separate cases depending on the cardinality of $V_{0}$. 


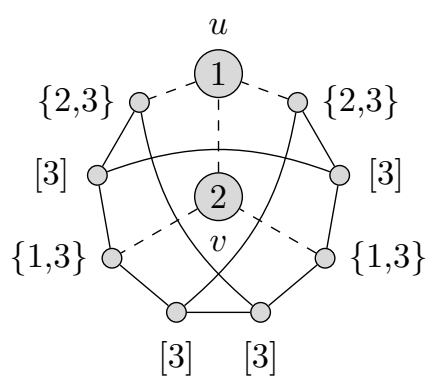

(a)

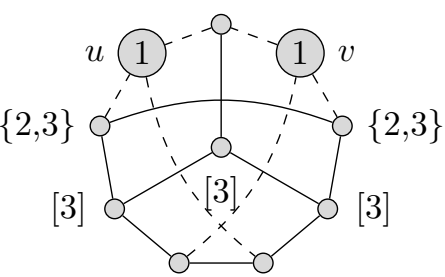

$\{2,3\}\{2,3\}$

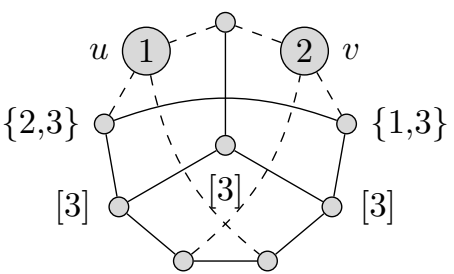

$\{1,3\}\{2,3\}$

(b)

Figure 5: $\left(G^{\prime}, L_{\phi}\right)$ from Case 1 of Theorem 25.

Case 1: $\left|\boldsymbol{V}_{\mathbf{0}}\right|=\mathbf{2}$, then $V_{0}=\{u, v\}$. Note that $G$ is distance transitive, thus up to an automorphism, there are only three cases to consider: (a) $\{u, v\} \in E(G), \phi(u)=1$ and $\phi(v)=2,(\mathrm{~b})\{u, v\} \notin E(G)$ and $\phi(u)=\phi(v)=1$, or (c) $\{u, v\} \notin E(G)$ and $\phi(u)=1$ and $\phi(v)=2$. In each case, it is easy to find a completion of $\phi$ (see Figure 5 (a)-(c) respectively).

Case 2: $\left|\boldsymbol{V}_{\mathbf{0}}\right|=\mathbf{3}$, then $\left|V\left(G^{\prime}\right)\right|=7$. Since any three vertices can share at most two edges among them, $\left|E\left(G^{\prime}\right)\right| \leqslant 8$. If $\left|E\left(G^{\prime}\right)\right| \leqslant 6$, then some component of $G^{\prime}$ is a tree, a contradiction to the maximality of $\left|V_{0}\right|$. Hence $\left|E\left(G^{\prime}\right)\right| \in\{7,8\}$. Note $G^{\prime}$ must contain a cycle, and thus must be connected and contain either $C_{5}$ or $C_{6}$ as induced subgraphs. It is straightforward to see that under these restrictions, $G^{\prime}$ must be one of the three graphs in Figure 6. For each of the graphs (b) and (c) in Figure 6, color the vertex marked $v$

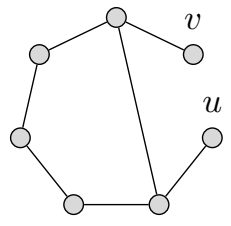

(a)

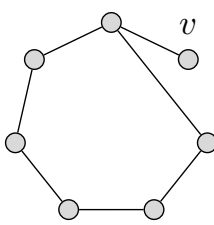

(b)

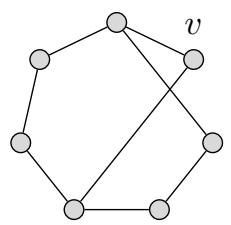

(c)

Figure 6: Possibilities for $G^{\prime}$ from Case 2 of Theorem 25.

any color available in its list, and update the lists on the neighborhood of $v$ in $G^{\prime}$. Delete $v$ from $G^{\prime}$ to obtain $G^{\prime \prime}=G^{\prime}-v \cong C_{6}$. What remains is a Hall 2-assignment $L_{\theta}$ that respects $L_{\phi}$, a contradiction to the maximality of $L_{\phi}$. Finally, for the graph shown in (a) of Figure 6, note the edge-count forces $G\left[V_{0}\right]$ to consist of vertices $V_{0}=\{a, b, c\}$ and single edge $\{a, b\}$. Because $G$ is edge-transitive, a simple case analysis yields only two possibilities, shown in Figure 7. Each has a completion, contradicting our choice of $\phi$. 

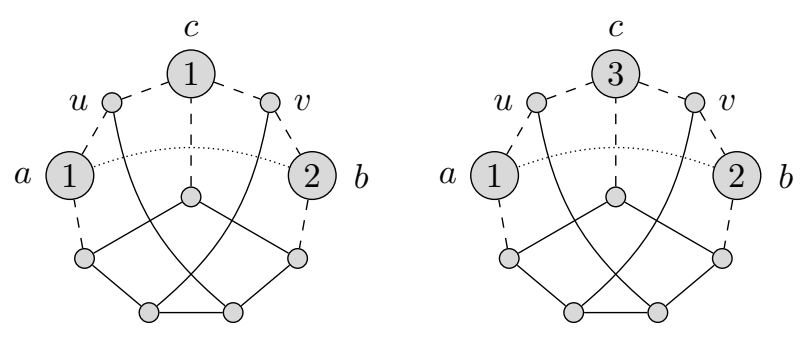

Figure 7: Possible pre-colorings that result in $G^{\prime}$ (solid edges) corresponding to graph (a) in Figure 6 from Case 2 of Theorem 25.

Case 3: $\left|\boldsymbol{V}_{\mathbf{0}}\right|=\mathbf{4}$, then $\left|V\left(G^{\prime}\right)\right|=6$. Any four vertices in $G$ can share at most 3 edges among them, so $\left|E\left(G^{\prime}\right)\right| \leqslant 6$. Therefore, we have two possibilities:

1. $G^{\prime}=C_{6}$. Here $L_{\phi}$ is a Hall 2-assignment to $G^{\prime}$, which is 2-choosable, hence $G^{\prime}$ is $L_{\phi}$-colorable, a contradiction.

2. $G^{\prime}$ is $C_{5}$ with a pendant edge. In this case we may color the vertex $v$ of degree one any color in its list, update the list on its only neighbor and delete $v$. What remains is a Hall 2-assignment $L_{\theta}$ to $C_{5}$ that respects $L_{\phi}$, a contradiction.

Case 4: $\left|\boldsymbol{V}_{\mathbf{0}}\right|=\mathbf{5}$, then $\left|V\left(G^{\prime}\right)\right|=5$. Any five vertices in $G$ can share at most 5 edges among them, so $\left|E\left(G^{\prime}\right)\right| \leqslant 5$. Therefore, $G^{\prime}=C_{5}$, which has Hall number 2 , and a similar contradiction is obtained.

Considerably more study of total Hall completable graphs is needed. Is there a nice characterization of total Hall completable graphs? Absent that, are there nontrivial necessary or sufficient conditions for a graph to be total Hall completable?

\section{Concluding Remarks}

Bobga et al. [2] posed the following three questions, two of which we addressed:

Question 1: Are there examples of graphs that are Hall $m$-completable but not Hall $(m+1)$-completable for some $m \geqslant 3$ ? In Section 2, we answered Question 1 in the affirmative.

Question 2: Let $G$ be a connected graph that is neither complete nor an odd cycle. Is it true that $G$ is Hall $\Delta(G)$-completable? We approached Question 2 in Section 3 by showing that bipartite graphs with Hall number no larger than three (e.g. bipartite planar graphs) are Hall $\Delta(G)$-completable. One may attempt to expand these techniques to investigate completability of graphs having "small" chromatic number (thus $k$-assignments with "small" $k$ are Hall assignments) and having "small" Hall number (thus Hall $k$ assignments with "small" $k$ are colorable). 
Question 3: If $G$ is a graph that is not Hall $m$-completable for some $m \geqslant \chi(G)$, but is Hall $(m+1)$-completable, is it possible that $G$ could fail to be Hall $(m+k)$-completable for some $k \geqslant 2$ ? We conjecture that the answer to this question is no.

Along the lines of Question 1, we ask the following:

Question 4: Are there examples of graphs that are Hall $m$-completable but not Hall $(m+1)$-completable for some $m>\chi(G)$ ? In all cases the authors are aware of, this behavior has only been observed when $m=\chi(G)$.

\section{Acknowledgements}

The authors thank Sibel Özkan and Peter Johnson for personal communication. The authors also thank the referees for their time and helpful comments.

\section{References}

[1] N. Alon and M. Tarsi. Colorings and orientations of graphs. Combinatorica, 12(2):125-134, 1992.

[2] B. B. Bobga, J. L. Goldwasser, A. J. W. Hilton, and P. D. Johnson, Jr. Completing partial Latin squares: Cropper's question. Australas. J. Combin., 49:127-151, 2011.

[3] P. Erdös, A. L. Rubin, and H. Taylor. Choosability in graphs. In Proceedings of the West Coast Conference on Combinatorics, Graph Theory and Computing (Humboldt State Univ., Arcata, Calif., 1979), Congress. Numer., XXVI, pages 125-157, Winnipeg, Man., 1980. Utilitas Math.

[4] C. Eslahchi and M. Johnson. Characterization of graphs with Hall number 2. J. Graph Theory, 45(2):81-100, 2004.

[5] A. J. W. Hilton and P. D. Johnson, Jr. Extending Hall's theorem. In Topics in combinatorics and graph theory (Oberwolfach, 1990), pages 359-371. Physica, Heidelberg, 1990.

[6] A. J. W. Hilton, P. D. Johnson, Jr., and E. B. Wantland. The Hall number of a simple graph. In Proceedings of the Twenty-seventh Southeastern International Conference on Combinatorics, Graph Theory and Computing (Baton Rouge, LA, 1996), volume 121, pages 161-182, 1996.

[7] V. G. Vizing. Coloring the vertices of a graph in prescribed colors. Diskret. Analiz, (29 Metody Diskret. Anal. v Teorii Kodov i Shem):3-10, 101, 1976.

[8] D. B. West. Introduction to graph theory. Prentice Hall Inc., Upper Saddle River, NJ, 1996. 